\title{
Measuring walking impairment in patients with intermittent claudication: Psychometric properties of the Walking Estimated-Limitation Calculated by History (WELCH) questionnaire
}

\author{
Farhad Rezvani ${ }^{\text {Corresp., } 1}$, Martin Härter ${ }^{1}$, Jörg Dirmaier ${ }^{1}$ \\ ${ }^{1}$ Department of Medical Psychology, Universitätsklinikum Hamburg-Eppendorf, Hamburg, Hamburg, Germany \\ Corresponding Author: Farhad Rezvani \\ Email address: f.rezvani@uke.de
}

Objectives. Patient-reported outcome measures can facilitate the assessment of walking impairment in peripheral artery disease patients with intermittent claudication in clinical trials and practice. The aim of this study was to test the psychometric properties of the German version of the 'Walking EstimatedLimitation Calculated by History' (WELCH) questionnaire.

Methods. The assessed properties included feasibility, test-retest reliability, construct validity (i.e., convergent, divergent and known-groups validity) and responsiveness using classic psychometric methods. Psychometric properties were tested as part of a randomized controlled home-based exercise trial for patients with symptomatic peripheral artery disease at Fontaine stage IIA/B.

Results. Analyses were conducted in subgroups of 1696 patients at baseline and 1233 patients at 12month follow-up (i.e., post-intervention) who completed the WELCH along with a battery of other selfreport measures. The WELCH did not exhibit relevant floor or ceiling effects $(<15 \%$ achieved lowest or highest possible scores), showed evidence for good test-retest reliability (ICC $=0.81,95 \% \mathrm{Cl}: 0.71$ 0.88 ) and was found to be well suited for self-completion by patients ( $<5 \%$ missing data per item). WELCH scores showed moderate to strong correlations with related measures of walking impairment at both time points (Walking Impairment Questionnaire: $r=.56-.74$; VascuQoL-25 activity subscale: $r=$ .61-. 66) and distinguished well among patients with poor and high quality of life when adjusting for confounders $(t=13.67, p<.001, d=.96)$. Adequate divergent validity was indicated by a weaker correlation between the WELCH and general anxiety at both time points (GAD-7: $r=-.14--.22$ ). The WELCH improved by 6.61 points (SD $=17.04,95 \% \mathrm{Cl}: 5.13-8.10, d=0.39)$ in response to exercise treatment and was able to identify large clinically important improvements observed on the walking distance (AUC $=.78,95 \% \mathrm{Cl}: .71-.84$ ) and speed subscales (AUC = .77, 95\% Cl: .68-.86) of the Walking Impairment Questionnaire.

Conclusions. The WELCH is considered a feasible, reliable and valid patient-reported outcome measure for the measurement of walking impairment in patients with peripheral artery disease. The WELCH showed evidence for responsiveness to changes in walking impairment, yet further studies are warranted to conclusively determine the WELCH's ability to detect intervention effects. 
1 Measuring walking impairment in patients with

2 intermittent claudication: Psychometric properties of

3 the Walking Estimated-Limitation Calculated by

4 History (WELCH) questionnaire

5

6 Farhad Rezvani ${ }^{1}$, Martin Härter, ${ }^{1}$, Jörg Dirmaier ${ }^{1}$

7

$8{ }^{1}$ Department of Medical Psychology, Universitätsklinikum Hamburg-Eppendorf, Hamburg,

9 Germany

10

11 Corresponding Author:

12 Farhad Rezvani, M.Sc.,

13 University Medical Center Hamburg Eppendorf, Department of Medical Psychology

14 Martinistrasse 52, 20246 Hamburg, Germany

15 Email address: $\underline{\text {.rezvani@uke.de }}$ 


\section{Abstract}

Objectives. Patient-reported outcome measures can facilitate the assessment of walking impairment in peripheral artery disease patients with intermittent claudication in clinical trials and practice. The aim of this study was to test the psychometric properties of the German version of the 'Walking Estimated-Limitation Calculated by History' (WELCH) questionnaire.

\section{Methods.}

The assessed properties included feasibility, test-retest reliability, construct validity (i.e., convergent, divergent and known-groups validity) and responsiveness using classic psychometric methods. Psychometric properties were tested as part of a randomized controlled home-based exercise trial for patients with symptomatic peripheral artery disease at Fontaine stage IIA/B.

Results. Analyses were conducted in subgroups of 1696 patients at baseline and 1233 patients at 12-month follow-up (i.e., post-intervention) who completed the WELCH along with a battery of other self-report measures. The WELCH did not exhibit relevant floor or ceiling effects $(<15 \%$ achieved lowest or highest possible scores), showed evidence for good test-retest reliability (ICC $=0.81,95 \% \mathrm{CI}: 0.71-0.88)$ and was found to be well suited for self-completion by patients $(<$ $5 \%$ missing data per item). WELCH scores showed moderate to strong correlations with related measures of walking impairment at both time points (Walking Impairment Questionnaire: $\mathrm{r}=.56$ -.74 ; VascuQoL-25 activity subscale: $\mathrm{r}=.61-.66$ ) and distinguished well among patients with poor and high quality of life when adjusting for confounders $(t=13.67, p<.001, d=.96)$. Adequate divergent validity was indicated by a weaker correlation between the WELCH and general anxiety at both time points (GAD-7: $r=-.14--.22$ ). The WELCH improved by 6.61 points ( $\mathrm{SD}=17.04,95 \% \mathrm{CI}: 5.13-8.10, \mathrm{~d}=0.39)$ in response to exercise treatment and was able to identify large clinically important improvements observed on the walking distance (AUC = $.78,95 \% \mathrm{CI}: .71-.84)$ and speed subscales $(\mathrm{AUC}=.77,95 \% \mathrm{CI}$ : .68-.86) of the Walking Impairment Questionnaire.

Conclusions. The WELCH is considered a feasible, reliable and valid patient-reported outcome measure for the measurement of walking impairment in patients with peripheral artery disease. The WELCH showed evidence for responsiveness to changes in walking impairment, yet further studies are warranted to conclusively determine the WELCH's ability to detect intervention effects. 


\section{Introduction}

51 Peripheral Artery Disease (PAD) is a global public health problem affecting an estimated 200

52 million people worldwide and has become one of the leading causes of disability and death over

53 recent decades. ${ }^{1-3}$ The most common symptom is intermittent claudication (IC), which refers to

54 cramping leg pain that is caused by exercise due to insufficient blood flow. 1,4

55

56

57

58

59

60

Rapid clinical assessment of walking impairment in symptomatic PAD patients is crucial in vascular surgery practice, providing a clinically relevant endpoint from the patient perspective by reflecting walking difficulties in everyday life and therefore considered a better tool for measuring patient-reported walking ability than functional surrogate endpoints. ${ }^{5}$ The "Walking Estimated-Limitation Calculated by History ( $(\mathrm{WELCH})$ questionnaire is a brief patient-reported outcome measure (PROM) instrument that requires minimal completion time for assessing walking capacity, with the intention to be used routinely in clinical practice. ${ }^{6}$ The WELCH has been translated and cross-validated into various languages ${ }^{6-10}$, has shown good feasibility results as it is easy to score and, compared to other PROMs, considered less prone to errors when selfadministered by the patient ${ }^{6,11}$, while correlating well with treadmill walking. ${ }^{6,10,12,13}$ To be proposed as a routine tool in the future, however, further external validation in larger samples and other languages are required. The purpose of the current study is, therefore, to psychometrically validate the WELCH in a German cohort of symptomatic PAD patients.

\section{Methods}

\section{Design}

The WELCH was validated as part of a prospective, randomized controlled trial (RCT) evaluating the effectiveness of a 12-month long home-based exercise program for patients with IC, PAD-TeGeCoach. The study protocol was registered and published elsewhere 
73 (ClinicalTrials.gov trial registration: NCT03496948). ${ }^{14}$ The study was conducted in accordance

74 with the Declaration of Helsinki and was approved by the ethics committee of the Medical Association of Hamburg (reference number: PV5708). All patients provided written informed consent.

\section{Study population}

Approximately 63000 PAD patients with IC symptoms aged 35-85 with a clinically confirmed

ICD-diagnosis of PAD at Fontaine stadium IIa (i.e., IC $>200$ meters) or IIb (i.e., IC $<200$

meters) within the last 36 months were identified using routinely collected health insurance data

from inpatient and outpatient encounters. Patients were excluded, if they had asymptomatic PAD within the last 12 months (Fontaine stadium I) or rest pain within the last 36 months (Fontaine stadium III or IV). As the diagnosis of PAD is often flawed, especially in outpatient settings, participants were interviewed about their IC symptoms prior to enrollment to verify the diagnosis of symptomatic PAD. A sample of 1982 PAD patients (recruitment rate approx. 3.2\%) were enrolled and randomized either into the exercise intervention (PAD-TeGeCoach) or the routine care group (see Figure 1 for RCT flow chart). 11 participants $($ TeGeCoach $n=10$; routine care $=$ 1) were withdrawn prematurely after randomization (data deletion request $n=1$, randomized without informed consent $n=1$, met exclusion criteria $n=8$, lack of verification of PAD diagnosis $n=1)$, leading to a final sample size of 1971 PAD patients $($ TeGeCoach $n=984$;

91 routine care $=987)$.

\section{Measures}

Only RCT data from measures relevant to the present study were used and are described in detail

94 elsewhere. ${ }^{14}$ While the WELCH was specified in the trial registry as a secondary outcome, the 95 authors failed to include it the study protocol. Notwithstanding this, the (internal and external) 
96

97

98

validity of the current study is not expected to be compromised as the trial was registered prospectively (i.e., before recruitment).

Participants received a battery of paper-based questionnaires by mail at each time point and were asked to return them using a prepaid envelope. To maximize return rates, participants who had not returned the questionnaire in time received a postal reminder after $2-4$ weeks. All participants were followed up at 12 months, irrespective of whether questionnaires had been returned at baseline. The participants could call the study team when they encountered problems completing the questionnaires.

\section{'Walking Estimated-Limitation Calculated by History' (WELCH) questionnaire}

The German version used in this study was requested and made available by the authors of the WELCH, which officially has not yet been psychometrically validated (see supplementary files). The WELCH was forward translated into German by a native-speaking health professional who was not a member of the WELCH development team, and was then closely back translated into French by the authors to ensure appropriate wording. After two rounds of forward and backwards translation, comparing the original and back-translated French versions, a version was reached that was considered acceptable by the authors. The WELCH consists of four items; items 1-3 are eight-point ordinal items, ranging from "impossible" to "3 hours or more", and assess the maximum duration that patients can maintain walking at different speeds in comparison to friends and relatives (i.e., slower/same/faster). Item 4 is a five-point ordinal item, ranging from "much slower" to "faster", and assesses the usual walking speed compared to friends and relatives. The WELCH score is generated by computing the sum of items 1-3, minus one, and multiplying it by the answer of item 4 , i.e. [(Item $1+$ Item $2+$ Item 3$)-1]$ x Item 4 . It is assumed that patients are able to walk at least 30 seconds at low speed so that the sum of the 
119 first three items is never 0. WELCH scores thus range from 0 (i.e., patient is able to walk for a

120 maximum of 30 seconds at slow speed) to 100 (i.e., patient is able to walk 3 hours or more at fast

121 speed). Missing values were handled as indicated by the WELCH authors; for items 1-3

122 (maximum walking duration), missing values were replaced by the mean of the other two

123 available items (i.e., mean imputation), whereas for item 4, missing values were automatically

124 replaced by $3 .{ }^{6}$ The German version of the WELCH can be found in the supplementary files.

125 Walking Impairment Questionnaire (WIQ)

126 The Walking Impairment Questionnaire (WIQ) is considered a reliable and valid instrument for

127 assessing walking impairment for different degrees of difficulty across three domains: walking

128 distance, walking speed and stair-climbing. ${ }^{15-18}$ WIQ scores are considered responsive to the

129 effect of treatment ${ }^{15,19}$, and are strongly correlated with maximum walking distance ${ }^{5,18}$,

130 objective measures of walking impairment ${ }^{16}$, as well as the ankle-brachial index. ${ }^{20}$ The

131 proportion of missing values was $<5 \%$ for all WIQ items. Item and scale-level missing data were

132 not imputed.

Kings College Vascular Quality of Life questionnaire (VascuQoL-25)

134 The VascuQoL-25 evaluates PAD-specific quality of life (QOL) and is divided into five

135 subscales: pain, symptoms, activities, social, and emotional. Although designed to measure

136 health-related QOL in PAD patients, all subscale scores and the composite score strongly

137 correlate with functional status outcomes. ${ }^{21,22}$ In addition, the activity subscale was suggested to

138 reflect physical functioning in patients with IC according to Wilson and Cleary's model for

139 health-related QOL ${ }^{23,24}$, and was therefore considered as a valid comparator instrument. The

140 VascuQoL-25 has also been shown to be responsive to changes in disease severity in PAD

Peer] reviewing PDF | (2021:02:58032:2:0:NEW 7 Jul 2021) 
141 patients. ${ }^{22,25}$ The proportion of missing values was $<5 \%$ for all VascuQoL-25 items and were 142 mean-imputed.

143 General Anxiety Disorder Scale (GAD-7)

144 The GAD-7 was shown to be a reliable and valid self-administered instrument with seven items

145 for screening general anxiety by measuring symptom severity in the last two weeks. ${ }^{26,27}$ Items

146 are scored from zero to three on a 4-point scale from 'not at all' to 'nearly every day'. A study

147 conducted in the German general population has found good psychometric properties for the

148 GAD-7. ${ }^{27}$ Item-level missing data were not imputed.

\section{Statistical analysis}

151 The psychometric properties of the WELCH were assessed in accordance with the standards set

152 out by the COSMIN group, which guide the development of rigorous methods to investigate

153 psychometric properties of PROMs ${ }^{28,29}$. When available, measurement properties were tested

154 against criteria proposed for good measurement properties of health status questionnaires ${ }^{30}$,

155 which were also used in a broad systematic review examining the psychometric validity of

156 PROMs for measuring IC. ${ }^{24}$

157 Floor and ceiling effects were assessed by examining frequency distributions of each item and

158 were considered to be present if $>15 \%$ of the study sample achieved the lowest or highest

159 possible score, respectively. ${ }^{30}$ Feasibility of the WELCH was assessed based on the number of

160 missing values in the study sample and per item before imputation. As a rule of thumb, multiple

161 imputation is not considered necessary and single imputation deemed sufficient, if the 
162 proportions of missing data are $<5 \%{ }^{31}$; therefore, the acceptable proportion of missing data for 163 each item was set at $<5 \%$.

164 The test-retest reliability of the WELCH was assessed based on a group of 67 PAD patients at 165 12-month follow-up who were instructed to fill out the questionnaire again after two weeks 166 under the same condition (i.e., self-completed at home and returned via mail), with patients 167 assumed to be stable during this time period without remembering their exact responses from two weeks earlier. To determine the level of consistency between these two time points (i.e., 169 stability of repeated measurements), the intraclass correlation coefficient (ICC) and the $95 \%$ 170 confidence interval (CI) were calculated using a two-way mixed effects model for single 171 measures with absolute reliability. Test-rest reliability of the WELCH was established when the 172 ICC is $>.70 .{ }^{24,30}$

173 Construct validity of the WELCH was verified with convergent validity. Pearson correlation 174 coefficients, with bootstrapped CIs based on $n=1000$ samples, were determined between the 175 WELCH and the comparator instruments at baseline and 12-month follow-up (i.e., WIQ, 176 VascuQoL-25 activity subscale), and were deemed satisfactory if there was a strong positive 177 correlation $\geq .50 .{ }^{24}$ Divergent validity was assessed by testing the association of the WELCH 178 with anxiety, a construct known to be unrelated to symptomatic PAD ${ }^{32}$; and was considered satisfactory if the correlation between the WELCH and the GAD-7 was weaker than with the 180 comparator instruments at baseline and 12-month follow-up. ${ }^{24}$ Known-groups validity was 181 determined using a t-test with degrees of freedom adjusted for unequal variances to examine the 182 extent to which the WELCH can significantly discriminate between PAD patients with poor to 183 moderate (VascuQoL-25 score $\leq 4$ ) and high QOL at baseline and 12-month follow-up, given 184 that QOL and walking impairment are known to be associated in patients with IC. ${ }^{33}$ The 
185 discriminatory ability of the WELCH was also assessed using a multiple linear regression to 186 control for potential confounders and mediators between QOL and WELCH scores. The model

187 was controlled for all significant variables reported in Table 1. The sample was assumed to be

188 highly representative of the population of IC patients, making the introduction of collider bias

189 unlikely. In the absence of defined quality criteria for health status questionnaires, known-groups

190 validity was also assessed using Cohen's d effect sizes $(0.2 \leq \mathrm{d}<.2 \leq$ small, $0.5 \leq \mathrm{d}<0.8$

191 medium, $\mathrm{d} \geq 0.8$ large).

192 Responsiveness of the WELCH was examined by testing the ability to distinguish patients who

193 have and have not changed after receiving the home-based exercise intervention (TeGeCoach),

194 using the area under the receiver operating characteristics (ROC) curve (AUC) at various

195 threshold settings for minimal clinically important differences (MCIDs) on the WIQ subscales ${ }^{34}$.

196 MCIDs reflect the health status change that patients consider beneficial. ${ }^{35}$ For the WIQ

197 subscales, these were previously determined based on an anchor-based method assessing

198 physical function quality of life following a 3-month (home or supervised) exercise intervention,

199 with exercise protocols closely resembling the PAD-TeGeCoach intervention used in this study

200 (i.e., intermittent walking to near maximal claudication pain while using an activity monitor

201 during exercise sessions). ${ }^{36,37}$ An $\mathrm{AUC}$ of $\geq .70$ was considered to indicate adequate

202 responsiveness. ${ }^{24,30}$ In addition, standardized effect sizes (d, baseline - 12-month follow-up)

203 were calculated for the WELCH and the convergent measures.

204 Analyses were performed using all available data at baseline and during 12-month follow-up.

205 Statistical analyses were performed using SPSS version 25 (IBM Corporation, Armonk, New

206 York, United States). Values of $p<.05$ (two-sided) were considered statistically significant.

207 Results 
208 Self-reported sociodemographic and clinical information of the PAD patients were collected at

209 both time points and are presented in Table 1, grouped by time point and subgroups used in the

210 analyses to allow tracking the pattern of missing data (see supplementary materials for an

211 extended version of Table 1). Of those enrolled $(\mathrm{N}=1971), 1696$ patients returned their

212 questionnaires at baseline (response rate: 86\%). 551 patients were lost to 12 -month follow-up,

213 while 1145 were followed up through 12-month follow-up. 88 patients returned their

214 questionnaire only at 12-month follow-up, resulting in a sample size of 1233 patients at 12-

215 month follow-up (response rate: 63\%). The response rates fall within the usual range of mail

216 surveys. ${ }^{38}$ Reasons for attrition were not identified, but may be attributed to the patient's right to

217 withdraw at any time without having to give a reason and without penalty. The sample sizes, per

218 analysis, using the RCT data are considered excellent for evaluating the psychometric properties

219 of the WELCH. ${ }^{39}$

220 Feasibility, and floor and ceiling effects

221 Score distributions and missing values per item before imputation are presented in Table 2. At

222 baseline, the total amount of missing data was $<5 \%$ per item. A total of $1611(95.0 \%)$ filled out

223 the WELCH completely at baseline, 79 patients (4.7\%) filled it out partially, while only 6

224 WELCH questionnaires were returned completely empty $(0.4 \%)$. The number of missing values

225 at 12-month follow-up was similarly low irrespective of study group (Table 2), indicating

226 excellent feasibility. In addition, the number of patients scoring the lowest or highest possible

227 score was $<15 \%$ in all items, indicating that there were no floor or ceiling effects irrespective of 228 study group. 
230 A group of 67 PAD patients filled out the WELCH twice within two weeks at 12-month follow-

231 up. The ICC for the WELCH score was 0.81 (95\% CI: $0.71-0.88)$, which indicates good test -

232 retest reliability.

\section{Construct validity}

234 Convergent and divergent validity analyses are presented in Table 3 . The findings indicate a

235 good convergent validity of the WELCH, as there was a strong positive correlation with the WIQ distance subscale (baseline: $\mathrm{n}=1564, \mathrm{r}=0.65,95 \% \mathrm{CI}: .62-.68, \mathrm{p}<.001 ; 12$ months: $\mathrm{n}=1$ $110, \mathrm{r}=0.70,95 \% \mathrm{CI}: .68-.73, \mathrm{p}<.001)$; a strong positive correlation with the WIQ speed subscale $(n=1575 r=0.68,95 \%$ CI: $.65-.71, p<.001 ; 12$ months: $n=1115, r=0.72,95 \%$ CI: .69 $-.75, \mathrm{p}<.001)$; a strong positive correlation with the WIQ stair climbing subscale $(\mathrm{n}=1$ $590, \mathrm{r}=0.56,95 \% \mathrm{CI}: .53-.59, \mathrm{p}<.001 ; 12$ months: $\mathrm{n}=1122, \mathrm{r}=0.60,95 \% \mathrm{CI}: .56-.63, \mathrm{p}<$ $.001)$; a strong positive correlation with the WIQ total score $(n=1472, r=0.70,95 \%$ CI: .68$.73, \mathrm{p}<.001 ; 12$ months: $\mathrm{n}=1037, \mathrm{r}=0.74,95 \% \mathrm{CI}: .72-.77, \mathrm{p}<.001$ ); and a strong positive correlation with the VascuQoL-25 activity scale $(\mathrm{n}=1660, \mathrm{r}=0.61,95 \%$ CI: $.58-.64, \mathrm{p}<.001$; 12 months: $\mathrm{n}=1$ 193, $\mathrm{r}=0.66,95 \% \mathrm{CI}: .63-.69, \mathrm{p}<.001)$. Furthermore, in absolute terms, there was a weaker correlation between the WELCH and the GAD-7 (baseline: $\mathrm{n}=1629, \mathrm{r}=$ 0.14, 95\% CI: $-.19--.09, \mathrm{p}<.001 ; 12$ months: $\mathrm{n}=1$ 162, $\mathrm{r}=-0.22,95 \% \mathrm{CI}:-.27--.16, \mathrm{p}<$ $.001)$, indicating adequate divergent validity. When separated by study group at 12 months of follow-up (TeGeCoach; routine care), the associations between the WELCH and the comparator instruments were nearly identical (see Table 3), demonstrating satisfactory construct validity 250 regardless of treatment status.

251 Poor to moderate QOL (VascuQoL-25 < 4; $\mathrm{n}=383 ; \mathrm{M}=14.95 ; \mathrm{SD}=12.20$ ) has been reported 252 by $23 \%$ of the sample at baseline. A significant mean score difference of 17.3 with a very large 
253 effect size $(\mathrm{d}=0.96 ; 95 \%$ CI: $0.84-1.01)$ was identified between PAD patients with high $(\mathrm{n}=1$

$254277 ; \mathrm{M}=32.23 ; \mathrm{SD}=19.38)$ and those with poor to moderate QOL, $\mathrm{t}(1001)=20.91, \mathrm{p}<.001$,

255 indicating excellent known-groups validity for the WELCH. In multiple linear regression

256 analysis, a logarithmic transformation was performed to correct for heteroscedasticity. Health-

257 related QOL remained a significant predictor of the WELCH score even after controlling for

258 potential confounders, $\mathrm{t}(847)=13.67, \mathrm{p}<.001$, which included age, BMI, income, comorbid

259 diseases, medication, gender, education, revascularization and heart rate training. The partial $\mathrm{r}$

260 for predicting WELCH score from health-related quality of life $(\mathrm{pr}=0.43)$ was not substantially

261 different from the zero-order Pearson's $r$ without controlling for confounders $(r=0.47)$. Similar

262 results were found at 12-month follow-up irrespective of study group (not reported).

\section{Responsiveness (TeGeCoach home-based exercise)}

264 From baseline to 12-month follow-up, the WELCH [range: 0-100] improved by 6.61 points (SD

$265=17.04,95 \%$ CI: $5.12-8.10, d=0.39)$ in the TeGeCoach group, from $28.86(\mathrm{SD}=18.98)$ to

$26635.47(\mathrm{SD}=22.29)$. During the same period, the WIQ distance, speed and stair climbing

267 subscale scores [range: $0-100]$ improved in the TeGeCoach group by $10.65(\mathrm{SD}=24.27,95 \%$

CI: $8.44-12.86, d=0.44), 6.83(\mathrm{SD}=19.55,95 \% \mathrm{CI}: 5.05-8.61, \mathrm{~d}=0.35)$ and 5.75 points $(\mathrm{SD}=$

$26920.51,95 \%$ CI: 3.90-7.60, $\mathrm{d}=0.28$ ), respectively. The WIQ total score [range: $0-100$ ] improved

270 by 7.66 points $(\mathrm{SD}=17.45,95 \% \mathrm{CI}: 5.96-9.37, \mathrm{~d}=0.44)$, and the VascuQOL [range: $0-7$ ] by

2710.32 points $(\mathrm{SD}=0.81,95 \% \mathrm{CI}: 0.25-0.39, \mathrm{~d}=0.40)$.

272 Figure 2 presents the ROC curves generated for the WELCH for small ( $+5 \%$ change), moderate

273 ( $+25 \%$ change) and large MCIDs ( $40 \%$ change) on the WIQ, and responsiveness statistics are

274 reported in detail in Table 4. The AUC for small changes was .66 ( $\mathrm{SE}=.02,95 \%$ CI: .62 - .71)

275 for the distance subscale, $.64(\mathrm{SE}=.03,95 \% \mathrm{CI}: .59-.69)$ for the speed subscale and $.65(\mathrm{SE}=$ 
$276.03,95 \%$ CI: .60 - .70) for the stair climbing subscale. For moderate changes, the AUC was .69

$277(\mathrm{SE}=.03,95 \% \mathrm{CI}: .64-.73)$ for the distance subscale, $.64(\mathrm{SE}=.03,95 \% \mathrm{CI}: .59-.69)$ for the

278 speed subscale and $.69(\mathrm{SE}=.04,95 \% \mathrm{CI}: .61-.77)$ for the stair climbing subscale. The AUC

279 for large changes was .78 $(\mathrm{SE}=.03,95 \% \mathrm{CI}: .71-.84)$ for the distance subscale, $.77(\mathrm{SE}=.05$,

$28095 \% \mathrm{CI}: .68-.86)$ for the speed subscale and $.68(\mathrm{SE}=.05,95 \% \mathrm{CI}: .58-.79)$ for the stair

281 climbing subscale. Another ROC curve has been generated to test the ability of the WELCH to

282 discriminate patients between study groups at 12-month follow-up (routine care vs. TeGeCoach).

283 This showed an AUC of .63 ( $\mathrm{SE}=.02,95 \% \mathrm{CI}: .59-.66)$.

\section{Discussion}

285 Further psychometric validation of PROMs that measure walking capacity in PAD patients is

286 required. This study sought to validate the German version of the WELCH, which was developed

287 to address the limitations of existing PROMs for the measurement of walking impairment in

288 PAD patients.

289 Consistent with previous findings ${ }^{6}$, few missing values and $>95 \%$ completely filled out

290 questionnaires largely support the excellent feasibility of the WELCH in PAD patients. The brief

291 nature of the questionnaire with only four items, and that it can be easily completed without

292 external support, makes it particularly attractive compared to other questionnaires, especially in

293 settings where time pressure is high (e.g. doctor's office). Furthermore, there were no floor or

294 ceiling effects, which enables the WELCH to discriminate equally between symptomatic PAD

295 patients across the entire IC severity spectrum.

296 In agreement with previous studies ${ }^{8,11}$, the WELCH has provided evidence for good

297 psychometric properties in terms of test-retest reliability. This finding is directly related to the 
298

299

300

301

302

303

304

305

306

307

308

309

310

311

312

313

314

315

316

317

318

319

320

usefulness of the WELCH in repeated measurement designs ensuring that scores changes are due to real changes rather than irrelevant artefacts, making an important contribution to its psychometric validity and reliability.

In terms of construct validity, results were in line with previous studies ${ }^{6,9,10}$, providing further evidence that the WELCH has good psychometric properties that make it suitable for use in assessing walking impairment living with intermittent claudication. As would be expected, the WELCH demonstrated satisfactory convergent validity, revealing a consistent pattern of moderator to strong correlations with the criterion measures. The associations between the WELCH and others PROMs indicate that the WELCH reflects similar but not identical constructs of walking impairment, with the WELCH showing the highest agreement with measures reflecting walking distance and walking speed (WIQ subscales). Notably, the correlation coefficients between the WELCH score and the WIQ subscales are fairly similar to those observed between the WELCH and treadmill maximum walking distance in previous studies, ${ }^{6,9,10}$ which further supports the validity of the WELCH for assessing walking impairment in symptomatic PAD patients. Simultaneously, the WELCH also shows a high correlation with health-related physical functioning (VascuQoL-25 activity subscale), indicating that the WELCH also quantifies the subjective patient experience by reflecting walking limitations in daily life. Furthermore, the WELCH was able to very accurately discriminate between patients with poor and high levels of health-related QOL, demonstrating excellent known-groups validity. Although the relationship between QOL and walking impairment is already well known ${ }^{33}$, these results indicate that the WELCH also indirectly reflects aspects of QOL in symptomatic PAD patients, further supporting the WELCH's value as a patient-relevant outcome measure by addressing the impact of PAD on those living with the disease. Finally, as 
321 predicted from previous findings ${ }^{32}$, the WELCH demonstrated good divergent validity in

322 relation to anxiety symptoms (GAD-7), likewise supporting the validity of the instrument.

323 To date, only few PROMs for PAD patients have been studied in terms their responsiveness,

324 which is a major shortcoming since PROMs are frequently used for measuring the effect of

325 treatment in research and clinical practice, ${ }^{24}$ raising doubts on the validity of results. Likewise,

326 evidence on the responsiveness of the WELCH is sparse. ${ }^{12}$ In agreement with the construct

327 validity results, the WELCH was found capable to detect large clinically important

328 improvements observed in walking distance and speed following a home-based training regimen,

329 suggesting that the WELCH may be considered responsive to exercise interventions, whereas

330 small to moderate improvements did not generate sufficient change on the WELCH. These

331 findings have direct implications for its use in therapy settings, as they show that the threshold

332 for detecting clinically important effects is relatively high when using the WELCH. The ability

333 to detect a restricted range of clinically meaningful changes $(+40 \%)$ in response to exercise

334 interventions may limit its utility in clinical settings, particularly since exercise interventions

335 generally have small to moderate, yet clinically meaningful effects on walking impairment. ${ }^{40,41}$

336 The WELCH may therefore be better suited to capture improvements after combined therapies of

337 IC (i.e., exercise therapy plus lower extremity revascularization), as greater improvements in

338 walking performance are usually achieved than after either therapy alone. ${ }^{42}$ The limited

339 responsiveness found here is also comparable to the responsiveness of the WELCH after

340 performing revascularization, where a moderate correlation with treadmill maximum walking

341 distance was shown. ${ }^{12}$ In addition, the 12-month time interval between the measurements may

342 have also reduced the WELCH's ability to detect improvements after exercise therapy, as the

343 WELCHs responsiveness tends to decrease over time. ${ }^{12}$ Despite the promising results reported, 
344 the responsiveness of the WELCH in relation to other walking impairment measures (i.e., other

345 PROMs, functional testing), and whether it depends on the time interval between measurements

346 (i.e., short-term, long-term change), mode of intervention (i.e., invasive, non-invasive) and

347 degree of change (i.e., small, medium, large intervention effects) still remains to be conclusively

348 determined in further studies.

349 The present study is the first study to evaluate the psychometric properties of the WELCH in a

350 German clinical population and has several strengths compared to previous validation studies.

351 With a patient to item ratio of 400:1, this is the largest validation study of the WELCH.

352 Furthermore, this is the first study to assess the psychometric properties of a PROM for assessing

353 IC based on the COSMIN checklist, which established guidelines for the psychometric validation

354 of health status PROMs ${ }^{30}$. Although assessing psychometric properties of health status PROMs

355 is common practice, the study quality in the field of PAD is often inadequate ${ }^{24}$, which underlines

356 the importance of adopting universal quality criteria. The COSMIN checklist provided a rigorous

357 methodological structure that helped in minimizing methodological bias. It would therefore be

358 useful to use the COSMIN checklist to further evaluate the measurement properties of PROMs

359 for PAD patients.

360 Several limitations of the study should be noted, including that the translation process did not

361 rigorously comply with the Principles of Good Practice for the Translation and Cultural

362 Adaptation Process for PROMs. ${ }^{43}$ Despite this shortcoming, the present study confirms the

363 psychometric validity of the German version of the WELCH, suggesting that the translation and

364 cultural adaptation process can be considered acceptable.

365 Furthermore, the comparator instruments used to test construct validity are not gold standards,

366 which may have reduced the observed correlations. Notwithstanding this, the WELCH showed 
367 high correlations with the comparator instruments, as expected, with the strongest associations

368 on the distance and speed subscales of the WIQ, supporting the validity of the WIQ in assessing

369 walking impairment ${ }^{16-19}$ and thus being well suited as a valid comparator instrument. To provide

370 further evidence for the construct validity of the WELCH, it should also be tested against third-

371 party assessments (e.g. ratings by health professionals) and gold standard measurements (e.g.

372 treadmill testing) in future validation studies.

\section{Conclusions}

374 This article provides evidence that the German version of the WELCH questionnaire is a valid

375 instrument for assessing walking impairment in patients with intermittent claudication. The

376 WELCH, when used appropriately, enables the assessment of walking impairment in PAD

377 patients, while compensating for the existing limitations of existing PROMs. In view of the

378 excellent feasibility and good construct validity, its use can be recommended in clinical settings,

379 as the medical team can quickly gain insight into the PAD patient's walking impairment

380 condition without the need for cumbersome and time-consuming functional assessments.

381 Nonetheless, despite its practicality, the WELCH should be treated with a degree of caution

382 when used to evaluate the benefits of exercise treatments in clinical trials and practice.

383 Alternatively, the WELCH merits consideration in vascular surgery to measure changes evoked

384 by combined treatments (i.e., exercise therapy plus lower extremity revascularization), which,

385 however, remains to be investigated in future studies.

\section{Acknowledgements}


387 We would like to thank the authors of the WELCH questionnaire, who kindly provided us with 388 the German version of the questionnaire. We would also like to thank Finja Mäueler, who 389 assisted us with great enthusiasm and commitment in the preparation of the manuscript.

\section{References}

1. Criqui MH, Aboyans V. Epidemiology of peripheral artery disease. Circ Res. 2015;116(9):1509-1526.

395

2. Sampson UK, Fowkes FG, McDermott MM, Criqui MH, Aboyans V, Norman PE, Forouzanfar MH, Naghavi M, Song Y, Harrell FE, Jr., Denenberg JO, Mensah GA, Ezzati M, Murray C. Global and regional burden of death and disability from peripheral artery disease: 21 world regions, 1990 to 2010. Glob Heart. 2014;9(1):145-158 e121.

3. Song P, Rudan D, Zhu Y, Fowkes FJI, Rahimi K, Fowkes FGR, Rudan I. Global, regional, and national prevalence and risk factors for peripheral artery disease in 2015: an updated systematic review and analysis. Lancet Glob Health. 2019;7(8):e1020-e1030.

4. Aboyans V, Ricco JB, Bartelink MEL, Bjorck M, Brodmann M, Cohnert T, Collet JP, Czerny M, De Carlo M, Debus S, Espinola-Klein C, Kahan T, Kownator S, Mazzolai L, Naylor AR, Roffi M, Rother J, Sprynger M, Tendera M, Tepe G, Venermo M, Vlachopoulos C, Desormais I, Group ESCSD. 2017 ESC Guidelines on the Diagnosis and Treatment of Peripheral Arterial Diseases, in collaboration with the European Society for Vascular Surgery (ESVS): Document covering atherosclerotic disease of extracranial carotid and vertebral, mesenteric, renal, upper and lower extremity arteriesEndorsed by: 
the European Stroke Organization (ESO)The Task Force for the Diagnosis and Treatment

410 of Peripheral Arterial Diseases of the European Society of Cardiology (ESC) and of the European Society for Vascular Surgery (ESVS). Eur Heart J. 2018;39(9):763-816.

5. Frans FA, Zagers MB, Jens S, Bipat S, Reekers JA, Koelemay MJ. The relationship of walking distances estimated by the patient, on the corridor and on a treadmill, and the Walking Impairment Questionnaire in intermittent claudication. J Vasc Surg. 2013;57(3):720-727 e721.

6. Ouedraogo N, Chanut M, Aubourg M, Le Hello C, Hidden V, Audat G, Harbonnier M, Abraham P. Development and evaluation of the Walking Estimated-Limitation Calculated by History questionnaire in patients with claudication. J Vasc Surg. 2013;58(4):981-988.

7. Abraham P, Godet R, Harbonnier M, Laneelle D, Leftheriotis G, Ouedraogo N. External Validation of the "Walking Estimated Limitation Calculated by History" (WELCH) Questionnaire in Patients with Claudication. Eur J Vasc Endovasc Surg. 2014;47(3):319325.

8. Cucato GG, Correia MdA, Farah BQ, Saes GF, Lima AHdA, Ritti-Dias RM, Wolosker N. Validation of a Brazilian Portuguese version of the walking estimated-limitation calculated by history (WELCH). Arq Bras Cardiol. 2016;106(1):49-55.

9. Abraham P, Godet R, Harbonnier M, Laneelle D, Leftheriotis G, Ouedraogo N. External validation of the "walking estimated limitation calculated by history" (WELCH) questionnaire in patients with claudication. Eur J Vasc Endovasc Surg. 2014;47(3):319325. 
431 10. Tew GA, Nawaz S, Humphreys L, Ouedraogo N, Abraham P. Validation of the English

432

433

434

435

436

437

438

439

440

441

442

443

444

445

446

447

448

449

450

451

452

453 version of the Walking Estimated-Limitation Calculated by History (WELCH)

questionnaire in patients with intermittent claudication. Vasc Med. 2014;19(1):27-32.

11. Ouedraogo N, Mahe G, Marchand J, Saïdi K, Leftheriotis G, Abraham P. Validation of a new simple questionnaire to "estimate ambulation capacity by history"(EACH) in patients with claudication. J Vasc Surg. 2011;54(1):133-138.

12. Henni S, Ammi M, Sempore Y, Hersant J, Zegar G, Gourdier AS, Picquet J, Abraham P. Treadmill Measured vs. Questionnaire Estimated Changes in Walking Ability in Patients With Peripheral Artery Disease. Eur J Vasc Endovasc Surg. 2019;57(5):676-684.

13. Fouasson-Chailloux A, Abraham P, Vielle B, Laporte I, Omarjee L, Ouedraogo N. The correlation of the "Walking Estimated-Limitation Calculated by History"(WELCH) questionnaire with treadmill maximal walking time is not impaired by age, in patients with claudication. Qual Life Res. 2015;24(8):1857-1864.

14. Rezvani F, Heider D, Härter M, König H-H, Bienert F, Brinkmann J, Herbarth L, Kramer E, Steinisch P, Freudenstein F. Telephone health coaching with exercise monitoring using wearable activity trackers (TeGeCoach) for improving walking impairment in peripheral artery disease: study protocol for a randomised controlled trial and economic evaluation. BMJ Open. 2020;10(6):e032146.

15. Regensteiner JG, Steiner JF, Panzer RJ, Hiatt WR. Evaluation of Walking Impairment by Questionnaire in Patients with Peripheral Arterial-Disease. Clin Res. 1990;38(2):A515A515.

16. McDermott MM, Liu K, Guralnik JM, Martin GJ, Criqui MH, Greenland P. Measurement of walking endurance and walking velocity with questionnaire: validation 
454

455

456

457

458

459

460

461

462

463

464

465

466

467

468

469

470

471

472

473

474

475

476

of the walking impairment questionnaire in men and women with peripheral arterial disease. J Vasc Surg. 1998;28(6):1072-1081.

17. Sagar S, Brown P, Zelt D, Pickett W, Tranmer J. Further clinical validation of the walking impairment questionnaire for classification of walking performance in patients with peripheral artery disease. Int J Vasc Med. 2012;2012.

18. Tew G, Copeland R, Le Faucheur A, Gernigon M, Nawaz S, Abraham P. Feasibility and validity of self-reported walking capacity in patients with intermittent claudication. $J$ Vasc Surg. 2013;57(5):1227-1234.

19. Nicolai SP, Kruidenier LM, Rouwet EV, Graffius K, Prins MH, Teijink JA. The walking impairment questionnaire: an effective tool to assess the effect of treatment in patients with intermittent claudication. J Vasc Surg. 2009;50(1):89-94.

20. Myers SA, Johanning JM, Stergiou N, Lynch TG, Longo GM, Pipinos, II. Claudication distances and the Walking Impairment Questionnaire best describe the ambulatory limitations in patients with symptomatic peripheral arterial disease. J Vasc Surg. 2008;47(3):550-555.

21. Morgan MB, Crayford T, Murrin B, Fraser SC. Developing the Vascular Quality of Life Questionnaire: a new disease-specific quality of life measure for use in lower limb ischemia. J Vasc Surg. 2001;33(4):679-687.

22. Mehta T, Venkata Subramaniam A, Chetter I, McCollum P. Assessing the validity and responsiveness of disease-specific quality of life instruments in intermittent claudication. Eur J Vasc Endovasc Surg. 2006;31(1):46-52.

23. Wilson IB, Cleary PD. Linking clinical variables with health-related quality of life. A conceptual model of patient outcomes. JAMA. 1995;273(1):59-65. 
477 24. Conijn AP, Jens S, Terwee CB, Breek JC, Koelemay MJ. Assessing the quality of available patient reported outcome measures for intermittent claudication: a systematic review using the COSMIN checklist. Eur J Vasc Endovasc Surg. 2015;49(3):316-334.

480

481

482

483

484 485

486

487

488

489

490

491

492

493

494

495

496

497

498

499

25. de Vries M, Ouwendijk R, Kessels AG, de Haan MW, Flobbe K, Hunink MG, van Engelshoven JM, Nelemans PJ. Comparison of generic and disease-specific questionnaires for the assessment of quality of life in patients with peripheral arterial disease. J Vasc Surg. 2005;41(2):261-268.

26. Spitzer RL, Kroenke K, Williams JB, Lowe B. A brief measure for assessing generalized anxiety disorder: the GAD-7. Arch Intern Med. 2006;166(10):1092-1097.

27. Lowe B, Decker O, Muller S, Brahler E, Schellberg D, Herzog W, Herzberg PY. Validation and standardization of the Generalized Anxiety Disorder Screener (GAD-7) in the general population. Med Care. 2008;46(3):266-274.

28. Mokkink LB, Terwee CB, Patrick DL, Alonso J, Stratford PW, Knol DL, Bouter LM, de Vet HC. The COSMIN study reached international consensus on taxonomy, terminology, and definitions of measurement properties for health-related patient-reported outcomes. $J$ Clin Epidemiol. 2010;63(7):737-745.

29. Mokkink LB, Prinsen CA, Patrick DL, Alonso J, Bouter LM, De Vet H, Terwee CB. COSMIN study design checklist for Patient-reported outcome measurement instruments. Department of Epidemiology and Biostatistics. 2019.

30. Terwee CB, Bot SDM, de Boer MR, van der Windt DAWM, Knol DL, Dekker J, Bouter LA, de Vet HCW. Quality criteria were proposed for measurement properties of health status questionnaires. J Clin Epidemiol. 2007;60(1):34-42.

31. Schafer JL. Multiple imputation: a primer. Stat Methods Med Res. 1999;8(1):3-15. 
500 32. Smolderen KG, Hoeks SE, Pedersen SS, van Domburg RT, de L, II, Poldermans D.

$501 \quad$ Lower-leg symptoms in peripheral arterial disease are associated with anxiety,

502 depression, and anhedonia. Vasc Med. 2009;14(4):297-304.

503 33. Golledge J, Leicht AS, Yip L, Rowbotham SE, Pinchbeck J, Jenkins JS, Clapperton R,

504 Dally-Watkins M, Singh MAF, Mavros Y. Relationship Between Disease Specific

505 Quality of Life Measures, Physical Performance, and Activity in People with Intermittent

506 Claudication Caused by Peripheral Artery Disease. Eur J Vasc Endovasc Surg. 2020.

507 34. Gardner AW, Montgomery PS, Wang M. Minimal clinically important differences in 508 treadmill, 6-minute walk, and patient-based outcomes following supervised and home-

509 based exercise in peripheral artery disease. Vasc Med. 2018;23(4):349-357.

510 35. Jaeschke R, Singer J, Guyatt GH. Measurement of health status: ascertaining the minimal 511 clinically important difference. Control Clin Trials. 1989;10(4):407-415.

512 36. Gardner AW, Parker DE, Montgomery PS, Blevins SM. Step-monitored home exercise 513 improves ambulation, vascular function, and inflammation in symptomatic patients with 514 peripheral artery disease: a randomized controlled trial. J Am Heart Assoc.

2015;3(5):e001107.

516 37. Gardner AW, Parker DE, Montgomery PS, Scott KJ, Blevins SM. Efficacy of quantified 517 home-based exercise and supervised exercise in patients with intermittent claudication: a 518 randomized controlled trial. Circulation. 2011;123(5):491-498.

519 38. Asch DA, Jedrziewski MK, Christakis NA. Response rates to mail surveys published in 520 medical journals. J Clin Epidemiol. 1997;50(10):1129-1136. 
521 39. Frost MH, Reeve BB, Liepa AM, Stauffer JW, Hays RD, Group MFPROCM. What is

522

523

524

525

526

527

528

529

530

531

532

533

534

535

536 sufficient evidence for the reliability and validity of patient-reported outcome measures? Value Health. 2007;10:S94-S105.

40. Golledge J, Singh TP, Alahakoon C, Pinchbeck J, Yip L, Moxon JV, Morris DR. Metaanalysis of clinical trials examining the benefit of structured home exercise in patients with peripheral artery disease. Br J Surg. 2019;106(4):319-331.

41. Parmenter BJ, Dieberg G, Smart NA. Exercise training for management of peripheral arterial disease: a systematic review and meta-analysis. Sports Med. 2015;45(2):231-244.

42. Biswas M, Capell WH, McDermott MM, Jacobs DL, Beckman JA, Bonaca MP, Hiatt WR. Exercise Training and Revascularization in the Management of Symptomatic Peripheral Artery Disease. Basic to Translational Science. 2021;6(2):174-188.

43. Wild D, Grove A, Martin M, Eremenco S, McElroy S, Verjee-Lorenz A, Erikson P, Translation ITFf, Cultural A. Principles of Good Practice for the Translation and Cultural Adaptation Process for Patient-Reported Outcomes (PRO) Measures: report of the ISPOR Task Force for Translation and Cultural Adaptation. Value Health. 2005;8(2):94-104. 
Figure 1

Flow chart of the study design. 


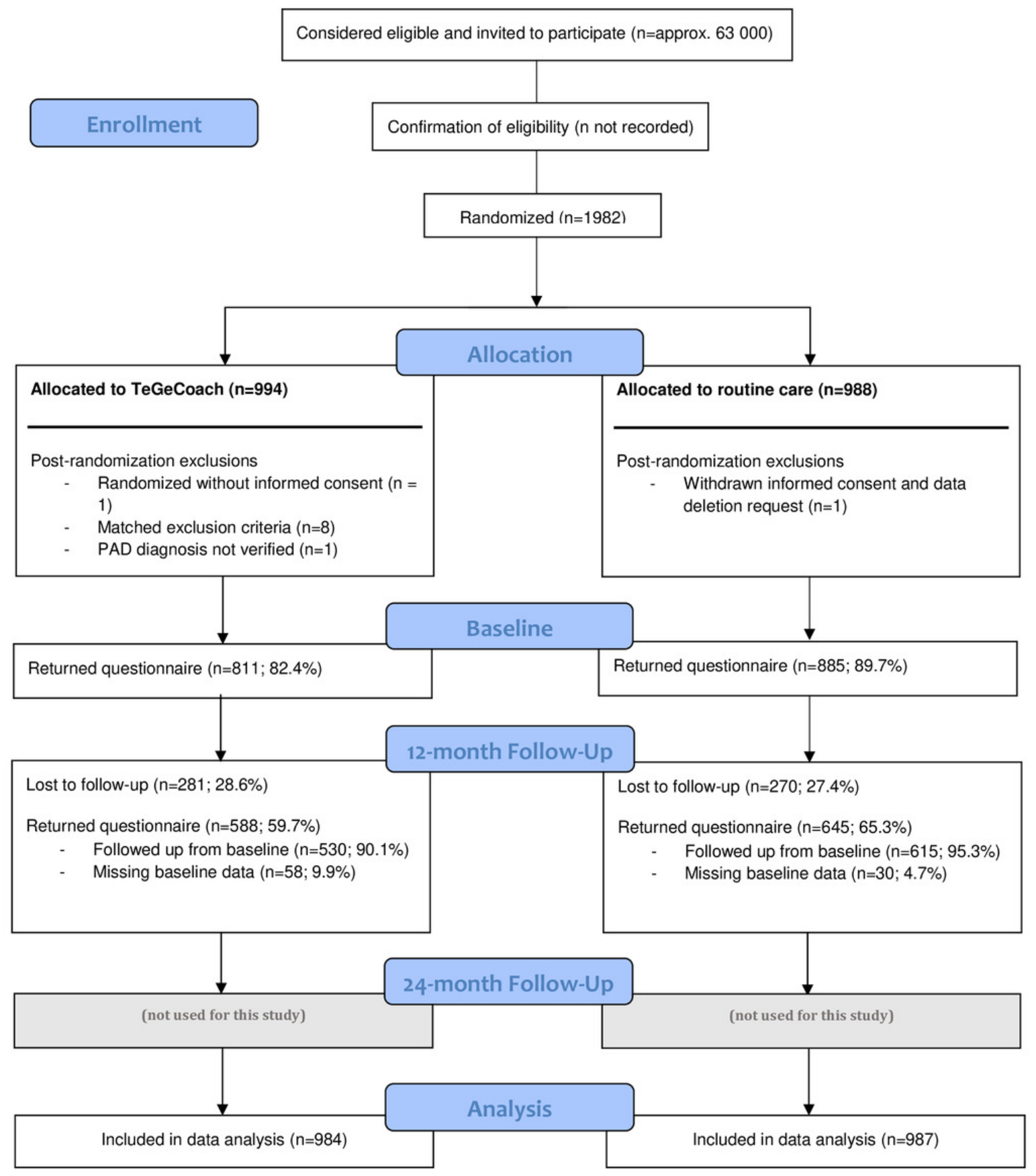




\section{Table $\mathbf{1}$ (on next page)}

Characteristics of subgroups used in the analyses (total sample at baseline, TeGeCoach group followed through to 12 months, TeGeCoach and routine care group at 12-month follow-up). 


\begin{tabular}{|c|c|c|c|c|}
\hline & \multirow{2}{*}{$\begin{array}{r}\text { Baseline } \\
\text { Total }(\mathrm{n}=1971)\end{array}$} & \multirow{2}{*}{\begin{tabular}{|c|c|}
$\begin{array}{c}\text { Followed through } \\
\text { to } 12 \text { months }\end{array}$ \\
TeGeCoach
\end{tabular}} & \multicolumn{2}{|c|}{ 12-month follow-up* } \\
\hline & & & TeGeCoach & Routine Care \\
\hline $\begin{array}{l}\text { N. of questionnaires received } \\
\text { Sex }{ }^{a}\end{array}$ & 1696 & 530 & 588 & 645 \\
\hline Female & $529(31.2)$ & $148(27.9)$ & $160(27.2)$ & $199(30.9)$ \\
\hline Male & $1146(67.6)$ & $371(70.0)$ & $417(70.9)$ & $441(68.4)$ \\
\hline No information provided & $21(1.2)$ & $11(2.1)$ & $11(1.9)$ & $5(0.8)$ \\
\hline Age (in years) ${ }^{b}$ & $66.3(8.6)$ & $67.0(8.2)$ & $67.1(8.3)$ & $67.3(8.4)$ \\
\hline Міпітит - Махітит & $35-81$ & $35-81$ & $35-81$ & $38-81$ \\
\hline$B M I^{b}$ & $28.1(5.0)$ & $27.9(5.4)$ & $27.8(5.3)$ & $28.0(4.7)$ \\
\hline Міпітит-Махітит & $15.0-75.8$ & $15.0-75.8$ & $14.5-75.8$ & $16.8-44.5$ \\
\hline \multicolumn{5}{|l|}{$\begin{array}{l}\text { Education }^{b} \text { (multiple choices } \\
\text { possible) }^{\text {ans }}\end{array}$} \\
\hline Apprenticeship & $1166(68.8)$ & $365(68.9)$ & $365(62.1)$ & $431(66.8)$ \\
\hline College & $562(33.1)$ & $177(33.4)$ & $177(30.1)$ & $224(34.7)$ \\
\hline University & $289(17.0)$ & $105(19.8)$ & $105(17.9)$ & $104(16.1)$ \\
\hline Other & $126(7.4)$ & $38(7.2)$ & $38(6.5)$ & $29(4.5)$ \\
\hline \multirow{2}{*}{\multicolumn{5}{|c|}{ Income $^{b}$}} \\
\hline & & & & \\
\hline$<500 €$ & $34(2.0)$ & $5(0.9)$ & $5(0.9)$ & $12(1.9)$ \\
\hline $500 €$ to $1000 €$ & $135(8.0)$ & $33(6.2)$ & $33(5.6)$ & $41(6.4)$ \\
\hline $1001 €$ to $1500 €$ & $215(12.7)$ & $58(10.9)$ & $58(9.9)$ & $69(10.7)$ \\
\hline $1501 €$ to $2000 €$ & $282(16.6)$ & $85(16.0)$ & $85(14.5)$ & $114(17.7)$ \\
\hline $2001 €$ to $2500 €$ & $306(18.0)$ & $108(20.4)$ & $108(18.4)$ & $103(16.0)$ \\
\hline $2501 €$ to $3000 €$ & $242(14.3)$ & $79(14.9)$ & $79(13.4)$ & $95(14.7)$ \\
\hline $3001 €$ to $3500 €$ & $147(8.7)$ & $42(7.9)$ & $42(7.1)$ & $67(10.4)$ \\
\hline $3501 €$ and more & $211(12.4)$ & $73(13.8)$ & $73(12.4)$ & $78(12.1)$ \\
\hline \multirow{2}{*}{\multicolumn{5}{|c|}{ Marital status ${ }^{b}$}} \\
\hline & & & & \\
\hline Single & $113(6.7)$ & $29(5.5)$ & $29(4.9)$ & $36(5.6)$ \\
\hline Married & $1088(64.2)$ & $366(69.1)$ & $366(62.4)$ & $412(63.9)$ \\
\hline Divorced/separated & $297(17.5)$ & $77(14.5)$ & $77(13.1)$ & $99(15.3)$ \\
\hline Widowed & $167(9.8)$ & $46(8.7)$ & $46(7.8)$ & $61(9.5)$ \\
\hline No information provided & $31(1.8)$ & $12(2.3)$ & $70(11.9)$ & $37(5.7)$ \\
\hline Number of children ${ }^{*} *$ & $1.7(1.1)$ & $1.8(1.1)$ & $1.8(1.1)$ & $1.7(1.2)$ \\
\hline Міпітит-Махітит & $0-11$ & $0-6$ & $0-6$ & $0-11$ \\
\hline \multicolumn{5}{|l|}{$\begin{array}{l}\text { Profession }{ }^{b} \text { (multiple choices } \\
\text { possible) }\end{array}$} \\
\hline Employed & $462(27.2)$ & $143(27.0)$ & $143(24.3)$ & $141(21.9)$ \\
\hline Unemployed & $77(4.5)$ & $15(2.8)$ & $15(2.6)$ & $21(3.3)$ \\
\hline Housewife/househusband & $61(3.6)$ & $14(2.6)$ & $14(2.4)$ & $32(5.0)$ \\
\hline Retired & $1057(62.3)$ & $351(66.2)$ & $351(59.7)$ & $410(63.6)$ \\
\hline Retired early & $52(3.1)$ & $23(4.3)$ & $23(3.9)$ & $12(1.9)$ \\
\hline $\begin{array}{l}\text { Permanently incapacitated for } \\
\text { work }\end{array}$ & $45(2.7)$ & $8(1.5)$ & $8(1.4)$ & $14(2.2)$ \\
\hline \multicolumn{5}{|l|}{$\begin{array}{l}\text { Diseases a (multiple choices } \\
\text { possible) }\end{array}$} \\
\hline Myocardial infarction & $217(12.8)$ & $79(14.9)$ & $85(14.5)$ & $82(12.7)$ \\
\hline Stroke & $149(8.8)$ & $51(9.6)$ & $53(9.0)$ & $53(8.2)$ \\
\hline Metabolism disorder & $965(56.9)$ & $314(59.2)$ & $344(58.5)$ & $383(59.4)$ \\
\hline Angina pectoris & $224(13.2)$ & $66(12.5)$ & $71(12.1)$ & $90(14.0)$ \\
\hline Lung disease & $271(16.0)$ & $71(13.4)$ & $79(13.4)$ & $117(18.1)$ \\
\hline Heart Failure & $259(15.3)$ & $80(15.1)$ & $87(14.8)$ & $101(15.7)$ \\
\hline Hypertension & $1225(72.2)$ & $371(70.0)$ & $409(69.6)$ & $482(74.7)$ \\
\hline Diabetes & $437(25.8)$ & $139(26.2)$ & $151(25.7)$ & $182(28.2)$ \\
\hline
\end{tabular}




\begin{tabular}{|c|c|c|c|c|}
\hline $\begin{array}{l}\text { Cancer } \\
\text { Drugs }{ }^{a} \text { (multiple choices } \\
\text { possible) }\end{array}$ & $155(9.1)$ & $51(9.6)$ & $55(9.4)$ & $64(9.9)$ \\
\hline Antihypertensive agents & $1253(73.9)$ & 397 (74.9) & $440(74.8)$ & $489(75.8)$ \\
\hline Platelet function inhibitor & $1370(80.8)$ & $444(83.8)$ & $491(83.5)$ & $540(83.7)$ \\
\hline Statins & $983(58.0)$ & $322(60.8)$ & $356(60.5)$ & 397 (61.6) \\
\hline \multicolumn{5}{|l|}{ Revascularization a } \\
\hline Yes & $499(29.4)$ & $182(34.3)$ & $192(32.7)$ & $185(28.7)$ \\
\hline No & $934(55.1)$ & $268(50.6)$ & $310(52.7)$ & $364(56.4)$ \\
\hline \multicolumn{5}{|l|}{ Group heart rate training a } \\
\hline Yes & $221(13.0)$ & $79(14.9)$ & $85(14.5)$ & $90(14.0)$ \\
\hline No & $1438(84.8)$ & $440(83.0)$ & $492(83.7)$ & $538(83.4)$ \\
\hline $\begin{array}{l}\text { No Information provided } \\
\text { Nationality }{ }^{a}\end{array}$ & $37(2.2)$ & $11(2.1)$ & $11(1.9)$ & $17(2.6)$ \\
\hline German & $1596(94.1)$ & $498(94.0)$ & $498(84.7)$ & $585(90.7)$ \\
\hline Other & $41(2.4)$ & $11(2.1)$ & $11(1.9)$ & $11(1.7)$ \\
\hline No information provided & $59(3.5)$ & $21(4.0)$ & $79(13.4)$ & $49(7.6)$ \\
\hline
\end{tabular}

a ${ }^{\text {NCategorical variables: } \mathrm{n}(\%)}$

2 ' 2 Quantitative variables: $\mathrm{M}$ (SD)

4 *Information on education, income, marital status, number of children, nationality and occupation was collected at baseline only, and was not available from the 88 participants 5 who completed only the 12-month follow-up questionnaire. These patients were included in no information provided. 


\section{Table 2 (on next page)}

Score distributions and missing values of WELCH items at baseline and 12-month followup. 


\begin{tabular}{|c|c|c|c|c|c|c|c|c|c|c|c|c|}
\hline & \multicolumn{6}{|c|}{ Baseline } & \multicolumn{6}{|c|}{ 12-month follow-up } \\
\hline & \multicolumn{2}{|c|}{ TeGeCoach $(n=811)$} & \multicolumn{2}{|c|}{ Routine care $(n=885)$} & \multicolumn{2}{|c|}{ Total $(\mathrm{n}=1696)$} & \multicolumn{2}{|c|}{ TeGeCoach $(n=588)$} & \multicolumn{2}{|c|}{ Routine care $(n=645)$} & \multicolumn{2}{|c|}{ Total $(\mathrm{n}=1233)$} \\
\hline & $\mathbf{n}$ & $\%$ & $\mathbf{n}$ & $\%$ & $\mathbf{n}$ & $\%$ & $\mathbf{n}$ & $\%$ & $\mathbf{n}$ & $\%$ & $\mathbf{n}$ & $\%$ \\
\hline \multicolumn{13}{|l|}{ Item 1} \\
\hline Impossible & 13 & 1.6 & 4 & 0.5 & 17 & 1.0 & 16 & 2.7 & 5 & 0.8 & 21 & 1.7 \\
\hline 30 seconds & 9 & 1.1 & 6 & 0.7 & 15 & 0.9 & 5 & 0.9 & 11 & 1.7 & 16 & 1.3 \\
\hline 1 minute & 27 & 3.3 & 25 & 2.8 & 52 & 3.1 & 10 & 1.7 & 21 & 3.3 & 31 & 2.5 \\
\hline 3 minutes & 98 & 12.1 & 112 & 12.7 & 210 & 12.4 & 43 & 7.3 & 78 & 12.1 & 121 & 9.8 \\
\hline 10 minutes & 239 & 29.5 & 240 & 27.1 & 479 & 28.2 & 118 & 20.1 & 160 & 24.8 & 278 & 22.5 \\
\hline 30 minutes & 204 & 25.2 & 234 & 26.4 & 438 & 25.8 & 133 & 22.6 & 155 & 24.0 & 288 & 23.4 \\
\hline 1 hour & 139 & 17.1 & 163 & 18.4 & 302 & 17.8 & 168 & 28.6 & 134 & 20.8 & 302 & 24.5 \\
\hline 3 hours or more & 60 & 7.4 & 79 & 8.9 & 139 & 8.2 & 73 & 12.4 & 59 & 9.1 & 132 & 10.7 \\
\hline Total & 789 & 97.3 & 863 & 97.5 & 1652 & 97.4 & 566 & 96.3 & 623 & 96.6 & 1189 & 96.4 \\
\hline NAs & 22 & 2.7 & 22 & 2.5 & 44 & 2.6 & 22 & 3.7 & 22 & 3.4 & 44 & 3.6 \\
\hline \multicolumn{13}{|l|}{ Item 2} \\
\hline Impossible & 22 & 2.7 & 17 & 1.9 & 39 & 2.3 & 11 & 1.9 & 14 & 2.2 & 25 & 2.0 \\
\hline 30 seconds & 19 & 2.3 & 19 & 2.1 & 38 & 2.2 & 9 & 1.5 & 20 & 3.1 & 29 & 2.4 \\
\hline 1 minute & 60 & 7.4 & 77 & 8.7 & 137 & 8.1 & 32 & 5.4 & 55 & 8.5 & 87 & 7.1 \\
\hline 3 minutes & 199 & 24.5 & 192 & 21.7 & 391 & 23.1 & 86 & 14.6 & 129 & 20.0 & 215 & 17.4 \\
\hline 10 minutes & 249 & 30.7 & 262 & 29.6 & 511 & 30.1 & 153 & 26.0 & 168 & 26.0 & 321 & 26.0 \\
\hline 30 minutes & 134 & 16.5 & 169 & 19.1 & 303 & 17.9 & 134 & 22.8 & 128 & 19.8 & 262 & 21.2 \\
\hline 1 hour & 82 & 10.1 & 96 & 10.8 & 178 & 10.5 & 106 & 18.0 & 78 & 12.1 & 184 & 14.9 \\
\hline 3 hours or more & 26 & 3.2 & 33 & 3.7 & 59 & 3.5 & 39 & 6.6 & 29 & 4.5 & 68 & 5.5 \\
\hline Total & 791 & 97.5 & 865 & 97.7 & 1656 & 97.6 & 570 & 96.6 & 621 & 96.3 & 1191 & 96.6 \\
\hline NAs & 20 & 2.5 & 20 & 2.3 & 40 & 2.4 & 18 & 3.1 & 24 & 3.7 & 42 & 3.4 \\
\hline \multicolumn{13}{|l|}{ Item 3} \\
\hline Impossible & 104 & 12.8 & 111 & 12.5 & 215 & 12.7 & 47 & 8.0 & 90 & 14.0 & 137 & 11.1 \\
\hline 30 seconds & 62 & 7.6 & 71 & 8.0 & 133 & 7.8 & 33 & 5.6 & 52 & 8.1 & 85 & 6.9 \\
\hline 1 minute & 145 & 17.9 & 143 & 16.2 & 288 & 17.0 & 72 & 12.2 & 90 & 14.0 & 162 & 13.1 \\
\hline 3 minutes & 216 & 26.6 & 217 & 24.5 & 433 & 25.5 & 125 & 21.3 & 144 & 22.3 & 269 & 21.8 \\
\hline 10 minutes & 149 & 18.4 & 194 & 21.9 & 343 & 20.2 & 139 & 23.6 & 131 & 20.3 & 270 & 21.9 \\
\hline 30 minutes & 78 & 9.6 & 79 & 8.9 & 157 & 9.3 & 86 & 14.6 & 70 & 10.9 & 156 & 12.7 \\
\hline 1 hour & 32 & 3.9 & 29 & 3.3 & 61 & 3.6 & 60 & 10.2 & 35 & 5.4 & 95 & 7.7 \\
\hline 3 hours or more & 5 & 0.6 & 17 & 1.9 & 22 & 1.3 & 6 & 1.0 & 7 & 1.1 & 13 & 1.1 \\
\hline Total & 791 & 97.5 & 861 & 97.3 & 1652 & 97.4 & 568 & 96.6 & 619 & 96.0 & 1187 & 96.3 \\
\hline NAs & 20 & 2.5 & 24 & 2.7 & 44 & 2.6 & 20 & 3.4 & 26 & 4.0 & 46 & 3.7 \\
\hline \multicolumn{13}{|l|}{ Item 4} \\
\hline Much slower & 120 & 14.8 & 131 & 14.8 & 251 & 14.8 & 62 & 10.5 & 93 & 14.4 & 155 & 12.6 \\
\hline Moderately slow & 320 & 39.5 & 362 & 40.9 & 682 & 40.2 & 182 & 31.0 & 245 & 38.0 & 427 & 34.6 \\
\hline A bit slower & 231 & 28.5 & 250 & 28.2 & 481 & 28.4 & 186 & 31.6 & 179 & 27.8 & 365 & 29.6 \\
\hline At the same speed & 107 & 13.2 & 112 & 12.7 & 219 & 12.9 & 125 & 21.3 & 90 & 14.0 & 215 & 17.4 \\
\hline Faster & 23 & 2.8 & 26 & 2.9 & 49 & 2.9 & 23 & 3.9 & 17 & 2.6 & 40 & 3.2 \\
\hline Total & 801 & 98.8 & 881 & 99.5 & 1682 & 99.2 & 578 & 98.3 & 624 & 96.7 & 1202 & 97.5 \\
\hline$N A s$ & 10 & 1.2 & 4 & 0.5 & 14 & 0.8 & 10 & 1.7 & 21 & 3.3 & 31 & 2.5 \\
\hline
\end{tabular}




\section{Table 3(on next page)}

Correlation between WELCH scores and other measures of walking impairment at baseline and 12-month follow-up. 


\begin{tabular}{|c|c|c|c|c|c|c|c|c|c|c|c|c|}
\hline \multirow[b]{3}{*}{$\begin{array}{l}\text { Correlation with WELCH } \\
\text { score }\end{array}$} & \multirow{2}{*}{\multicolumn{3}{|c|}{ Baseline }} & \multicolumn{9}{|c|}{ 12-month follow-up } \\
\hline & & & & \multicolumn{3}{|c|}{ TeGeCoach } & \multicolumn{3}{|c|}{ Routine care } & \multicolumn{3}{|c|}{ Total } \\
\hline & $\mathbf{n}$ & $\mathbf{r}$ & $\begin{array}{r}\text { Bootstrapped } \\
95 \% \text {-CI }\end{array}$ & $\mathbf{n}$ & $\mathbf{r}$ & $\begin{array}{r}\text { Bootstrapped } \\
95 \% \text {-CI }\end{array}$ & $\mathbf{n}$ & $\mathbf{r}$ & $\begin{array}{r}\text { Bootstrapped } \\
95 \% \text {-CI }\end{array}$ & $\mathrm{n}$ & $\mathbf{r}$ & $\begin{array}{r}\text { Bootstrapped } \\
95 \% \text {-CI }\end{array}$ \\
\hline WIQ walking distance & 1564 & .65 & $.62-.68$ & 532 & .71 & $.67-.74$ & 578 & .69 & $.64-.73$ & 1110 & .70 & $.68-.73$ \\
\hline WIQ walking speed & 1575 & .68 & $.65-.71$ & 531 & .73 & $.69-.76$ & 584 & .71 & $.67-.75$ & 1115 & .72 & $.69-.75$ \\
\hline WIQ stair climbing & 1590 & .56 & $.53-.59$ & 535 & .60 & $.55-.64$ & 587 & .60 & $.54-.64$ & 1122 & .60 & $.56-.63$ \\
\hline WIQ total & 1472 & .70 & $.68-.73$ & 493 & .75 & $.72-.79$ & 544 & .73 & $.69-.76$ & 1037 & .74 & $.72-.77$ \\
\hline VascuQoL Activity & 1660 & .61 & $.58-.64$ & 571 & .67 & $.63-.71$ & 622 & .64 & $.60-.68$ & 1193 & .66 & $.63-.69$ \\
\hline$G A D-7$ & 1629 & -.14 & $-.19--.09$ & 559 & -.22 & $-.28--.14$ & 603 & -.21 & $-.28--.13$ & 1162 & -.22 & $-.27-. .16$ \\
\hline
\end{tabular}




\section{Table 4 (on next page)}

WELCH responsiveness statistics at various thresholds reflecting small, moderate and large changes on the WIQ subscales 


\begin{tabular}{llllllllll}
\hline & \multicolumn{3}{l}{ Small $(+5 \%$ change $)$} & \multicolumn{4}{l}{ Moderate $(+25 \%$ change $)$} & \multicolumn{3}{l}{ Large $(+40 \%$ change $)$} \\
\hline $\begin{array}{l}\text { WIQ } \\
\text { subscale }\end{array}$ & $\begin{array}{l}\text { Walking } \\
\text { Distance }\end{array}$ & $\begin{array}{l}\text { Walking } \\
\text { Speed }\end{array}$ & $\begin{array}{l}\text { Stair } \\
\text { climbing }\end{array}$ & $\begin{array}{l}\text { Walking } \\
\text { Distance }\end{array}$ & $\begin{array}{l}\text { Walking } \\
\text { Speed }\end{array}$ & $\begin{array}{l}\text { Stair } \\
\text { climbing }\end{array}$ & $\begin{array}{l}\text { Walking } \\
\text { Distance }\end{array}$ & $\begin{array}{l}\text { Walking } \\
\text { Speed }\end{array}$ & $\begin{array}{l}\text { Stair } \\
\text { climbing }\end{array}$ \\
\hline Threshold $^{a}$ & $\geq 5$ & $\geq 2$ & $\geq 12$ & $\geq 15$ & $\geq 11$ & $\geq 35$ & $\geq 42$ & $\geq 37$ & $\geq 41$ \\
\hline AUC & 0.66 & 0.64 & 0.65 & 0.69 & 0.64 & 0.69 & 0.78 & 0.77 & 0.68 \\
\hline SE & 0.02 & 0.03 & 0.03 & 0.03 & 0.03 & 0.04 & 0.03 & 0.05 & 0.05 \\
\hline $95 \%$ CI & $0.62-0.71$ & $0.59-0.69$ & $0.60-0.70$ & $0.64-0.73$ & $0.59-0.69$ & $0.61-0.77$ & $0.71-0.84$ & $0.68-0.86$ & $0.58-0.79$ \\
\hline$n$ positive & 249 & 256 & 183 & 170 & 175 & 36 & 41 & 26 & 25 \\
\hline$n$ negative & 257 & 250 & 323 & 336 & 331 & 470 & 465 & 480 & 481 \\
\hline
\end{tabular}

1 a adopted from Gardner et al., 2018.

2 
Figure 2

WELCH ROC curves for small, moderate and large changes on the WIQ subscales.

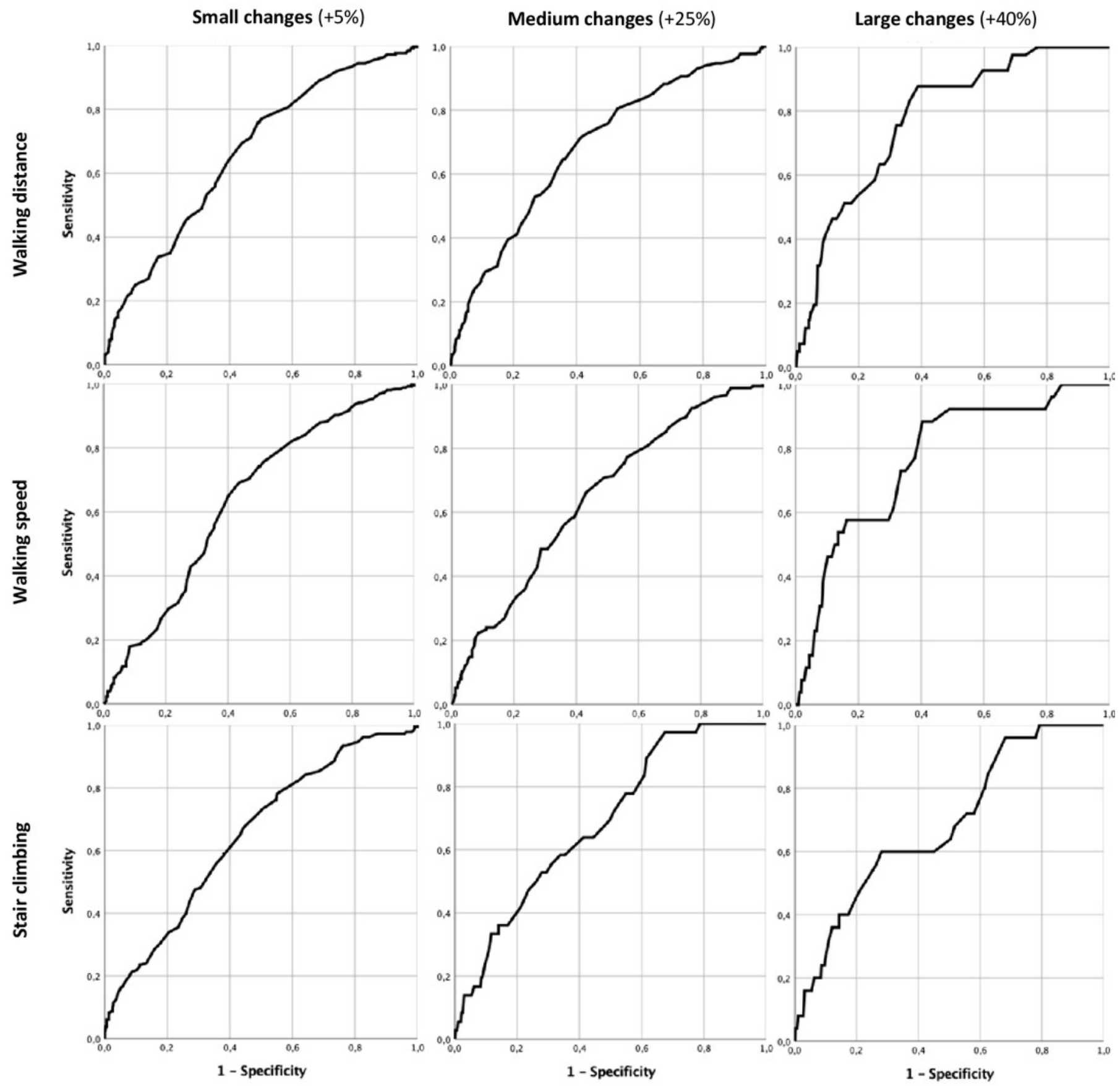

